\section{(6) \\ OPEN ACCESS}

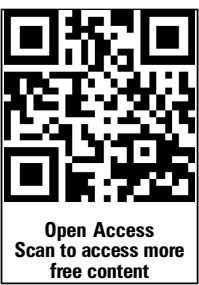

Handling editor Tore K Kvien

- Additional material is published online only. To view please visit the journal online (http://dx.doi.org/10.1136/ annrheumdis-2013-203901)

${ }^{1}$ Center for Stem Cell and Tissue Engineering, School of Medicine, Zhejiang University, Hangzhou, Zhejiang, China

${ }^{2}$ Zhejiang Provincial Key Lab for Tissue Engineering and Regenerative Medicine, School of Medicine, Zhejiang University, Hangzhou, Zhejiang, China

${ }^{3}$ Department of Orthopaedics, The Second Affiliated Hospital of Wenzhou Medical University, Wenzhou, China

${ }^{4}$ Department of Biosystems Science \& Engineering, ETHZurich, Mattenstrasse, Switzerland

${ }^{5}$ Department of Biochemistry and Molecular Biology, Schoo of Medicine, Zhejiang University, Hangzhou, Zhejiang, China

\section{Correspondence to} Professor Hong Wei Ouyang or Professor Yiting Zhou, Center for Stem Cell and Tissue Engineering, School of Medicine, Zhejiang Provincial Key Lab for tissue engineering and regenerative medicine, Zhejiang University, $866 \mathrm{Yu}$ Hang Tang Road, Hangzhou, Zhejiang 310058, China; hwoy@zju.edu.cn

Received 7 May 2013 Revised 21 August 2013 Accepted 26 October 2013 Published Online First 20 November 2013

\section{CrossMark}

To cite: Zhu S, Lu P, Liu $\mathrm{H}$, et al. Ann Rheum Dis 2015:74:285-293.

\title{
Inhibition of Rac1 activity by controlled release of NSC23766 from chitosan microspheres effectively ameliorates osteoarthritis development in vivo
}

\author{
Shouan Zhu, ${ }^{1,2}$ Ping Lu, ${ }^{1,2}$ Huanhuan Liu, ${ }^{1,2}$ Pengfei Chen, ${ }^{1,2}$ Yan Wu, ${ }^{1,2}$ \\ Yanyan Wang, ${ }^{1,2}$ Heng Sun, ${ }^{1,2}$ Xiaolei Zhang, ${ }^{1,2,3}$ Qingqing Xia, ${ }^{1,2}$ Boon Chin Heng, ${ }^{4}$ \\ Yiting Zhou, ${ }^{1,5}$ Hong Wei Ouyang ${ }^{1,2}$
}

\section{ABSTRACT}

Background Osteoarthritis (OA) is a degenerative joint disease characterised by cartilage degradation and chondrocyte hypertrophy. A recent study showed that Rac1 promoted expression of MMP13 and chondrocyte hypertrophy within the growth plate. These findings warrant further investigations on the roles of Rac1 in OA development and therapy in animal models.

Objective To investigate the role and mechanistic pathway of Rac1 involvement in pathological changes of $\mathrm{OA}$ chondrocytes in vitro and $\mathrm{OA}$ development in vivo, as well as to develop a strategy of modulating Rac1 activity for $\mathrm{OA}$ treatment.

Material and methods $\mathrm{OA}$ and normal cartilage from human or mice were used for immunohistochemical study and Rac1 activity assay. Chondrocytes treated with IL $1 \beta$ and the untreated control were subjected to the Rac1 activity assay. Chondrocytes transfected with CA-Rac1, DN-Rac1 or GFP were cultured under conditions for inducing calcification. To evaluate the effect of Rac1 in OA development, an OA model was created by anterior cruciate ligament transection in mice. CA-Rac1, DN-Rac1 and GFP lentivirus, or NSC23766, were injected intra-articularly. Joints were subjected to histological analysis.

Results It was found that there is aberrant Rac1 activation in human OA cartilage. Rac1 activity could also be elevated by IL1 $\beta$. Additionally, activated Rac1 promoted expression of MMP13, ADAMTS-5 and COLX by chondrocytes, partially through the $\beta$-catenin pathway. Moreover, activation of Rac1 in knee joints by CA-Rac1 lentivirus accelerated OA progression, while inhibition of Rac1 activity by DN-Rac1 lentivirus or Rac1 inhibitor NSC23766 delayed OA development. Therefore, we developed a strategy of controlled release of NSC23766 from chitosan microspheres to OA joints, which effectively protected cartilage from destruction. Conclusions These findings demonstrated that Rac1 activity is implicated in OA development. Also, controlled release of Rac1 inhibitor is a promising strategy for $\mathrm{OA}$ treatment.

\section{INTRODUCTION}

Osteoarthritis (OA) is the most commonly occurring degenerative joint disease that lacks functional pharmacological treatment. In OA chondrocytes, the hypertrophy or matrix degradation-related genes including COL10A1 (collagen, type X, a1), ${ }^{1} 2$ MMP13 (matrix metallo-peptidase-13), ${ }^{3}$ ADAMTS-5 (a disintegrin and metalloproteinase with thrombospondin motifs 5$)^{4} 5$ and Runx2 (runt-related transcription factor- 2$)^{6}$ are upregulated. This, in turn, leads to cartilage-specific extracellular matrix degradation and disruption of cartilage homeostasis. Hence, the study of protein molecules regulating hypertrophy, or matrix degradation-related genes, is important for developing effective therapeutics for OA.

Recent studies ${ }^{7-12}$ from different groups reveal the important roles of small GTPases in governing chondrocyte development, hypertrophy and maturation during endochondral bone formation. Rac1, one of small GTPases is required for chondrocyte condensation mediated by $\mathrm{N}$-cadherin and acts as a positive regulator of chondrogenesis and chondrocyte hypertrophy. ${ }^{7}$ The regulatory effect of Rac1 on chondrocyte differentiation was verified by genetically modified mice. In vivo, Rac1-deficient growth plates displayed delayed ossification, reduced chondrocyte proliferation and increased apoptosis, ${ }^{9}$ partly due to reduced mitogenic activity through Rac1-iNOS-NO signalling. ${ }^{10}$ Similar results were also observed in limb bud development. ${ }^{11} 12$ As Rac1 plays important roles in the physical hypertrophy and ossification of growth plate chondrocytes during bone formation, it is logical to investigate whether Rac1 is also implicated in pathological hypertrophy and ossification of articular chondrocyte in OA joints. A very recent study by Long and colleagues demonstrated that Rac1 was required for fibronectin fragment-mediated MMP13 production by articular chondrocytes in vitro. ${ }^{13}$ Therefore, this warrants further study on the relationship between Rac1 and OA development in vivo, as well as developing a new OA treatment strategy through modulation of Rac1 activity.

We hypothesise that Rac1 activity has a significant relationship with OA development. Inhibition of Rac1 activity appears to be a promising strategy for OA treatment. Therefore, chondrocytes were cultured under conditions for inducing hypertrophy and calcification, and the effect and mechanistic pathway of Rac1 involvement in chondrocyte hypertrophy and calcification were investigated in vitro. The role of Rac1 activity in OA development in vivo was investigated with mice OA knee joints. 


\section{METHODS AND MATERIALS}

\section{Human cartilage and chondrocytes}

Human OA cartilage was obtained from patients undergoing total knee replacement surgery. Control normal cartilage was obtained postmortem from human subjects with no history of OA. The patient's consent, as well as approval of the local ethics committee were obtained prior to harvesting of human tissue samples. Human articular chondrocytes were harvested by overnight incubation of $1 \mathrm{~mm}^{2}$ cartilage slices with $2 \mathrm{mg} / \mathrm{mL}$ of collagenase $\mathrm{P}$ in Dulbecco's modified Eagle medium (DMEM) supplemented with $10 \%$ fetal bovine serum and $40 \mu \mathrm{g} / \mathrm{mL}$ gentamicin at $37^{\circ} \mathrm{C}$. After resuspension and filtration through a $0.7 \mu \mathrm{m}$ filter, cells were cultured in a 24 -well plate at a seeding density of $2 \times 10^{5}$ cells $/ \mathrm{mL}$.

\section{Protein extraction from human cartilage and mice joints}

Human cartilage or mice joints were first dissected into small pieces $(0.5 \mathrm{~mm} \times 0.5 \mathrm{~mm})$ with a knife, and then milled in $200 \mu \mathrm{L}$ radioimmune precipitation assay (RIPA) lysis buffer with protease inhibitor cocktail and phenylmethanesulfonyl fluoride with a homogeniser (Ultra-Turrax IKA T10 basic). The mixture was then centrifuged at $10000 \mathrm{~g}$, with the supernatant being collected and used for Rac1 activity assay.

\section{Pull-down assay for Rac1 activity}

Rac1 activation assays were performed by using a commercially available Rac1 activation assay kit (Thermo Pierce \#16118), in accordance with the manufacturer's protocol.

\section{Western blot analysis}

Cellular protein was extracted with RIPA lysis buffer, and the protein concentration was determined with a bicinchoninic acid assay kit (Pierce \#23227). The extracted cellular protein was loaded on SDS-PAGE-denaturing gels. After electrophoresis, proteins were transferred to a polyvinylidene difluoride membrane and blocked in 5\% bovine serum albumin for $1 \mathrm{~h}$ at room temperature. The membrane was incubated overnight at $4^{\circ} \mathrm{C}$ with anti-Rac1 or anti-glyceraldehyde-3-phosphate dehydrogenase (GAPDH) antibody. After washing in Tris-buffered saline with Tween (TBST), horseradish peroxidase (HRP) secondary antibody was diluted 1:1000 in 5\% bovine serum albumin (BSA) solution and incubated with the membrane for $1 \mathrm{~h}$ at room temperature. Excess secondary antibody was rinsed off the membrane with TBST, and a chemiluminiscent signal was generated by using western blot detection reagents (ECL, Beyotime Institute of Biotechnology) according to the manufacturer's protocol.

\section{Lentivirus transfection}

Chondrocytes were transfected with lentivirus when cells were $30-50 \%$ confluent at a multiplicity of infection of $200 ; 12 \mathrm{~h}$ after infection, more than $95 \%$ of the cells were still viable and the culture medium was then changed. Three days later, all transfected cells were passaged for use in further experiments.

\section{Chondrocyte calcification in vitro}

Cells were trypsinised and seeded into a 24 -wells plate, and calcification was induced for 2 weeks with calcification medium, composed of DMEM supplemented with 1\% ITS+ (BD Biosciences, Franklin Lakes, New Jersey, USA), 1\% antibiotic-antimycotic solution, $50 \mu \mathrm{g} / \mathrm{mL}$ ascorbate-2-phosphate (Sigma, St Louis, Missouri, USA), $40 \mu \mathrm{g} / \mathrm{mL}$ L-proline (Sigma), $100 \mathrm{nM}$ dexamethasone (Sigma) and $1 \mathrm{nM}$ triiodothyronine (T3) (Sigma). Rac1 inhibitor, NSC23766 $(50 \mu \mathrm{M})$ was added to the calcification medium to inhibit Rac1 activity during induction of chondrocyte hypertrophy.

\section{Real-time PCR}

The mRNA expression levels of genes associated with hypertrophy (MMP13, ADAMTS-5, COLX and Runx2) by human chondrocytes transfected with CA-Rac1, DN-Rac1 or GFP were assessed with real-time PCR. Total cellular RNA was isolated by lysis in TRIzol (Invitrogen, Carlsbad, California, USA). PCR was performed using Brilliant SYBR Green QPCR Master Mix (TakaRa) with a Light Cycler apparatus (ABI 7900HT). The amplification efficiencies of primer pairs were validated to enable quantitative comparison of gene expression. All primer sequences (Invitrogen) were designed using primer V.5.0 software. Each QPCR was performed on at least three different experimental samples, and representative results are presented as target gene expression normalised to the reference gene GAPDH. Error bars represent 1 SD from the mean of technical replicates. The following primer sequences were used: MMP13 sense 5'-ATGCAGTCTTTCTTCGGCTTAG-3', antisense 5'-ATGCCATCGTGAAGTCTGGT-3'; ADAMTS-5 sense 5'-ATCACCCAATGCCAAGG-3', antisense 5'-AGCAGAGTAGGAGACAAC-3'; Runx2 sense 5'-GTGATAAATTCAGAAGGGAGG-3', antisense $5^{\prime}$-CTTTTGCTAATGC TTCGTGT-3'; COLX sense 5'-GTGTTTTACGCTGAACGATACCAA-3', antisense 5'-ACCTGGTTTCCCTACAGCTGATG-3'.

\section{Immunocytochemical staining}

Human chondrocytes cultured in F12 medium supplemented with $10 \%$ fetal bovine serum, were fixed in $4 \%$ formalin solution for $15 \mathrm{~min}$ and permeabilised with $0.3 \%$ Triton X-100 in phosphate-buffered saline (PBS) for $10 \mathrm{~min}$. The fixed cells were then incubated with primary antibodies against ADAMTS-5 (Abcam, ab41037), FLAG (Beyotime Institute of Biotechnology, \#AF519), MMP13 (Santa Cruz, sc-30073), COLX (Abcam, ab58632), $\beta$-catenin (CST, \#9582) followed with goat antimouse or goat antirabbit secondary antibodies conjugated with Alexa Fluor 488 or Alexa Fluor 546 flourescent dyes (Invitrogen). Finally, the cell nuclei were visualised by DAPI (Beyotime Institute of Biotechnology) and viewed under confocal microscopy (Olympus, BX61W1-FV1000, Japan).

\section{Animal OA model}

For all experiments with induced OA, C57L/6 mice were used. All animal experiments were approved by the ethics committee of Zhejiang University. OA was induced by anterior cruciate ligament transection. OA pathology developed gradually throughout 8 weeks. Rac1 inhibitor, NSC23766 (30 $\mathrm{g} / \mathrm{site}$, TOCRIS, \#2161) dissolved in PBS was administered as an intra-articular injection three times a week. Hyaluronic acid (HA) with Chitosan microspheres encapsulated with NSC23766 were injected into OA joints once a week. Knee joints were isolated at week 4, 6 and 8 and processed for histological analysis. Joints from week 4 were also used for protein extraction and Rac1 activity assay. Intra-articular injections of Lentivirus were carried out at 10, 25 and 40 days post OA surgery, and knee joints were collected at 6 weeks post-OA surgery for histological analysis, immunohistochemical staining and protein extraction. All animals were treated according to standard guidelines approved by the Zhejiang University Ethics Committee (no. ZJU2013105002).

\section{Histological analysis and immunohistochemistry of murine knee joints and human cartilage}

The isolated knee joints were processed for histology and immunohistochemistry. Tissues were fixed in $4 \%(\mathrm{v} / \mathrm{v})$ neutral 
buffered formalin for $24 \mathrm{~h}$, and decalcified in neutral $10 \%$ EDTA solution for 1 month at room temperature. Subsequently, the samples were dehydrated through an alcohol gradient, cleared, and embedded in paraffin blocks. Histological sections $(8 \mu \mathrm{m})$ were then prepared using a microtome, and six representative sections of each joint from various depths were mounted on slides and subsequently stained with Safranin Orange. The stained sections were photographed digitally under a microscope. Human cartilage sections $(8 \mu \mathrm{m})$ were prepared using freezing microtome.

Subsequently, human cartilage sections and murine joint sections were incubated overnight with either polyclonal mouse anti-Rac1 antibody (Thermo Pierce \#16118), mouse anti-FLAG antibody (Beyotime Institute of Biotechnology), rabbit anti-COLX antibody (Abcam, ab58632) or rabbit anti-ADAMTS-5 antibody (Abcam, ab41037). This was followed by incubation with goat antimouse secondary antibodies conjugated with HRP (Beyotime Institute of Biotechnology) or with Alexa Fluor 488 or Alexa Fluor 546 flourescent dyes (Invitrogen). The stained specimens were photographed digitally and viewed under confocal microscopy (Olympus, BX61W1-FV1000, Japan).

\section{Preparation of chitosan microspheres}

Chitosan microspheres were prepared through the water-in-oil $(\mathrm{W} / \mathrm{O})$ emulsion solvent diffusion method. Chitosan solution $(2 \% \mathrm{w} / \mathrm{v})$ was prepared by dissolving chitosan (Shanghai Bio Science and Technology) in $2.5 \%(\mathrm{v} / \mathrm{v})$ acetic acid aqueous solution (Sinopharm Chemical Reagent) at room temperature. The chitosan solution was mixed with inhibitor by stirring overnight with a magnetic stirrer to produce a homogeneous mixture. Then, $5 \mathrm{~mL}$ of the mixture was aspirated into a syringe pump, and then added drop-wise into the oil phase $(24.72 \mathrm{~mL})$ consisting of $14 \mathrm{~mL}$ liquid paraffin (Sinopharm Chemical Reagent), $10 \mathrm{~mL}$ petroleum ether, $0.72 \mathrm{~mL}$ Span 80 (Sangon Biotech) at a flow rate of $4 \mathrm{~mL} / \mathrm{h}$ with continuous stirring at $1500 \mathrm{rpm}$. A syringe needle with $0.2 \mathrm{~mm}$ internal diameter was used for this process. After the solvent diffusion procedure, the suspension was cross-linked using 25\% (v/v) glutaraldehyde solution as a cross-linking agent. Addition of the cross-linker was carried out three times at time intervals of $15 \mathrm{~min}$, with the following volumes of glutaraldehyde: $0.64,0.64$ and $0.32 \mathrm{~mL}$. Subsequently, the suspension was stirred at room temperature for cross-linking, and then centrifuged at $3000 \mathrm{rpm}$ for $5 \mathrm{~min}$, followed by discarding the supernatant fluid. The microspheres were then washed several times with petroleum ether (three times) (Sinopharm Chemical Reagent), methanol (two times) (Sinopharm Chemical Reagent), acetone (one time) (Sinopharm Chemical Reagent), Isopropyl alcohol (one time) (Sinopharm Chemical Reagent), ethanol (one time) and distilled water (three times). After washing, the microspheres were collected by lyophilising with a freeze dryer to remove residual water. For the control group, pure chitosan microspheres were also prepared by directly dropping chitosan solution into the oil phase under the same conditions.

\section{Statistical analysis}

All quantitative data are presented as mean \pm SD. Non-parametric statistical tests were performed to assess statistically significant differences in the data between groups. Values of $\mathrm{p}<0.05$ were considered to be statistically significant. Significance level was presented as either $* \mathrm{p}<0.05$ or $* \mathrm{p}<0.01$.

\section{RESULTS}

\section{Aberrant activation of Rac1 in human OA chondrocytes}

To evaluate if there is aberrant Rac1 activation in OA, we compared Rac1 expression and activity by immunohistochemical analysis and pull-down assay in human OA cartilage obtained from subjects undergoing total knee replacement, as well as normal cartilage samples from trauma subjects. We found that total Rac1 was highly expressed in both OA and normal cartilage of mice and humans (figure 1A). However, the activated form of Rac1 (Rac1-GTP) was significantly upregulated only in
Figure 1 Rac1 expression and activity in osteoarthritis (OA) and normal articular cartilage. (A) Immunohistochemistry assay of total Rac1 expression in human, mice $O A$ and normal cartilage. Scale bars, $50 \mu \mathrm{m}$. (B) Rac1 pull-down assay of Rac1-GTP level in human OA and normal cartilage. (C) Rac1 pull-down assay of Rac1-GTP level when human primary chondrocytes treated with IL1 $\beta$ demonstrated by western blot and measurement of relative density of Rac1-GTP band. ${ }^{*} p<0.05,{ }^{*} p<0.01$.
A

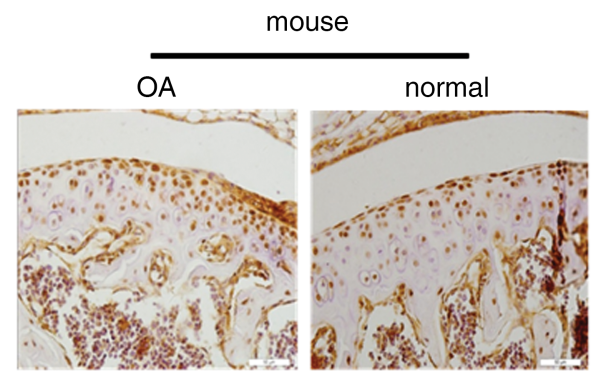

B

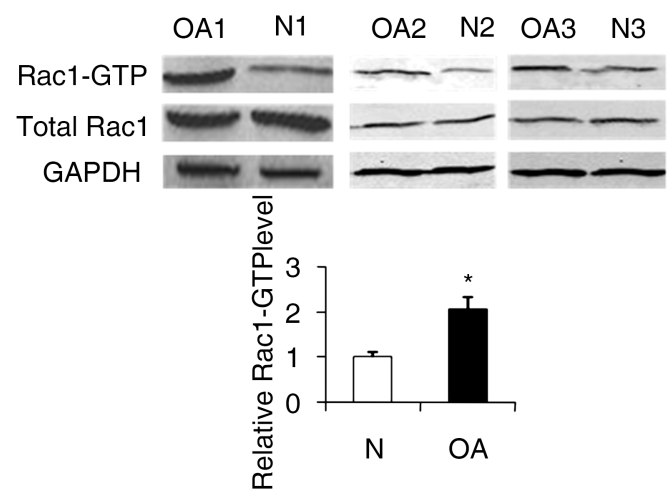

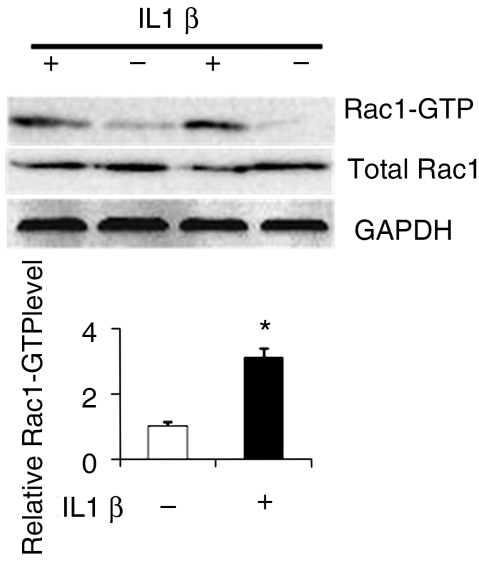


OA cartilage (figure 1B). We subsequently investigated whether Rac1 could be activated by pro-inflammatory factor IL1 $\beta$ in human articular chondrocytes primary culture system. As shown in figure 1C, in chondrocytes treated with IL1 $\beta$, Rac1-GTP level was greatly elevated (figure 1C). These data indicated that the elevation of Rac1 activity is a characteristic event during human OA development. This prompted us to investigate the roles of activated Rac1 in chondrocyte pathological change.

Activated Rac1 enhances chondrocyte hypertrophy and expression of matrix degradation-related genes, as well as calcification in vitro
We subsequently investigated whether activated Rac1 predisposes OA phenotype. Accordingly, we introduced constitutively active Rac1 mutant (CA-Rac1) expression within OA chondrocytes and measured the levels of hypertrophy and matrix degradation-related genes. CA-Rac1 expression resulted in higher expression of MMP13 ( $p=0.056)$, ADAMTS-5 $(p=0.022)$, COLX $(p=0.058)$ and Runx2 $(p=0.021)$ (figure 2A,B). Conversely, dominant negative Rac1 (DN-Rac1) reduced hypertrophy and expression of matrix degradation-related genes. We then used an in vitro chondrocyte calcification model that can be visualised by Alizarin Red Staining and quantified by an OD value of $405 \mathrm{~nm}$. Direct activation of Rac1 by CA-Rac1 resulted
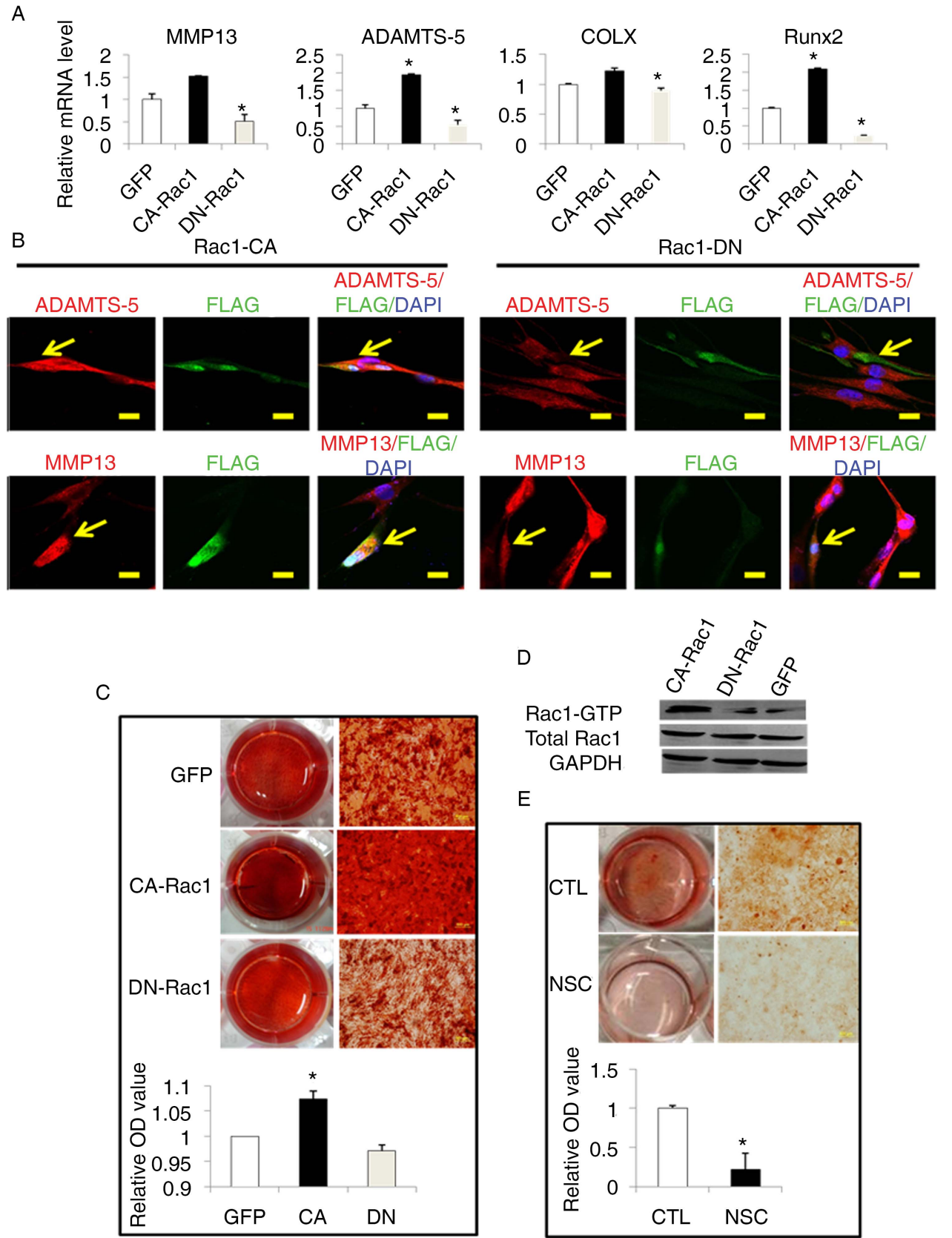

Figure 2 Modulation of Rac1 activity in chondrocytes controlled hypertrophy-related genes expression and chondrocytes calcification. (A and B) Effect of CA-Rac1 and DN-Rac1 on MMP13, ADAMTS-5, COLX and Runx2 expression demonstrated by mRNA (A) and protein (B), arrowed chondrocytes were successfully transfected with ectopic Rac1 (green) and displayed different genes expression showed by different fluorescence intensity compared with neighbouring chondrocytes. Values are means $\pm S D(n=3)$. ${ }^{*} p<0.05,{ }^{*} p<0.01$. Scale bars, $30 \mu \mathrm{m}$. (C and $\left.D\right)$ Effect of Rac1 inhibition by DN-Rac1 or activation by CA-Rac1 (C) on chondrocytes calcification visualised by Alizarin Red staining (D). (E) Effect of Rac1 inhibition by NSC23766 on chondrocytes calcification. Values are means \pm SD $(n=3)$. ${ }^{*} p<0.05,{ }^{*} p<0.01$. Scale bars, $100 \mu \mathrm{m}$. 
in notably enhanced calcification, while inhibition of Rac1 activity by DN-Rac1 led to decreased formation of calcium nodule (figure 2C,D). Similarly, pharmacological inhibition of Rac1 by NSC23766 also resulted in significantly decreased chondrocyte calcification (figure 2E).

\section{Inhibition of Rac1 activity in articular chondrocytes delays OA development}

Evidence for the pathological role of activated Rac1 in vitro prompted us to analyse the functional relevance of Rac1 inhibition to the development of OA in a mouse model. We first investigated whether Rac1 inhibition prevents OA phenotype in vivo. DN-Rac1, CA-Rac1 and GFP lentivirus were injected intra-articularly into mice OA joints. Ectopic expression of these constructs was detected by immunohistochemical assay. Rac1 pull-down assay demonstrated that Rac1 activity in articular chondrocytes was successfully modulated by lentivirus injection (figure 3A). The GFP control group showed similar OA development with a rough cartilage surface and decreased Safranin Orange staining (figure $3 \mathrm{~B}$ and figure S1) compared with

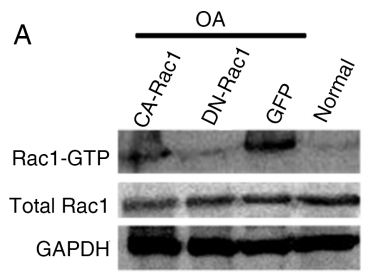

C

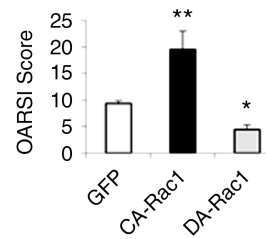

B

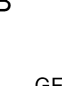

GFP
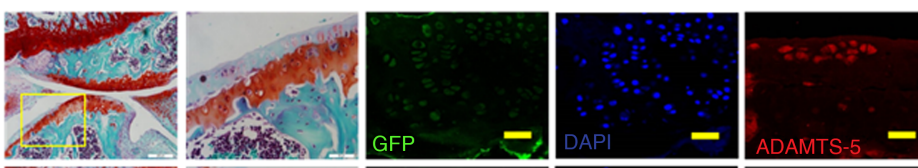

CA-Rac
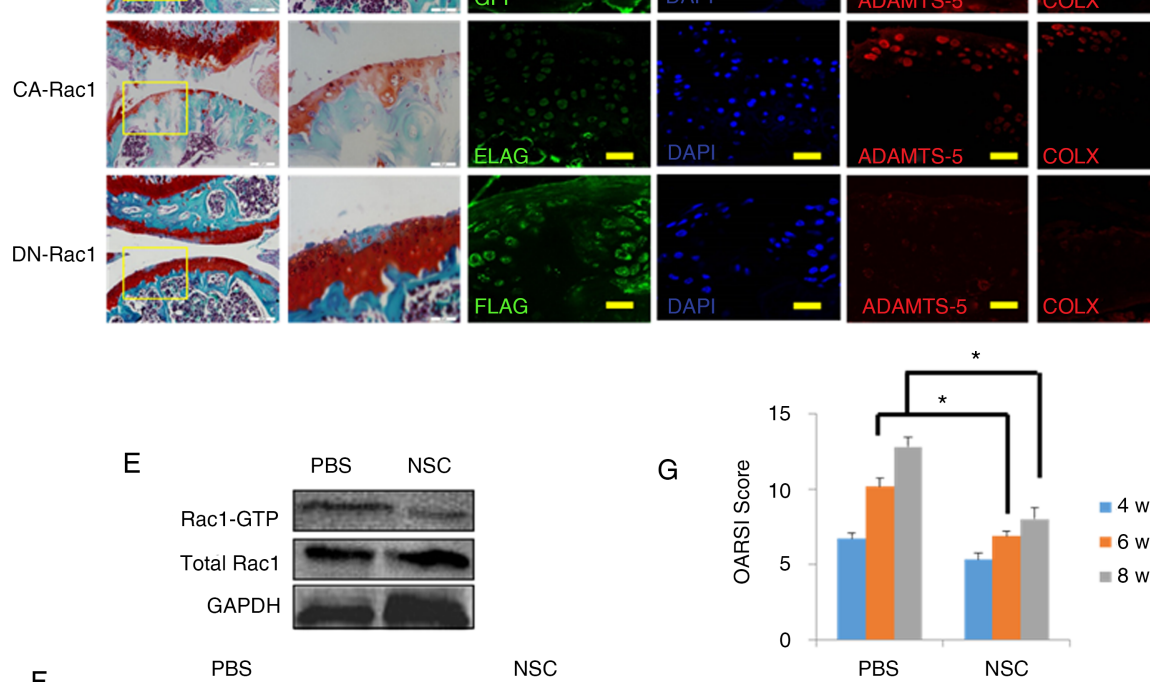

F

E

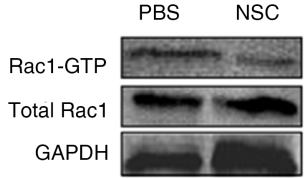

PBS

$10 x$

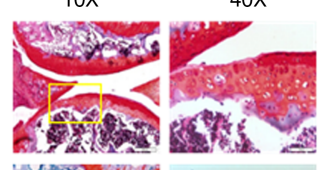

$6 w$

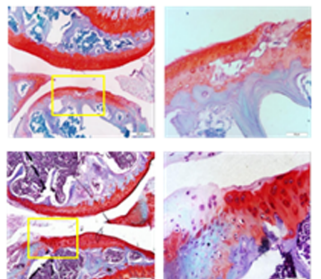

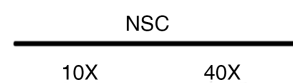
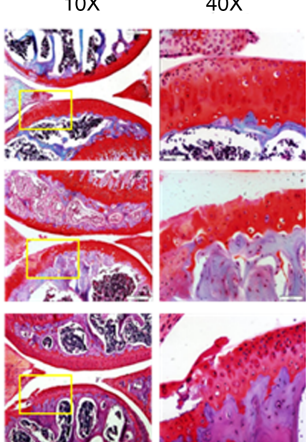

$H$
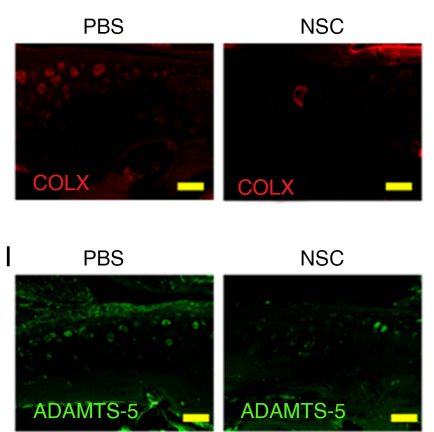

NSC

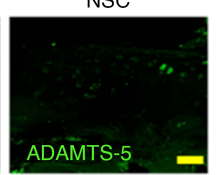

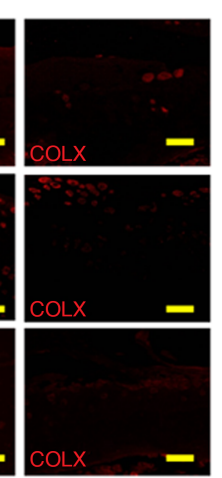
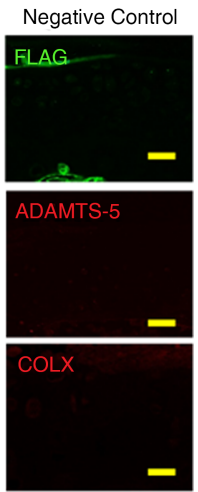

$4 w$

$6 \mathrm{w}$
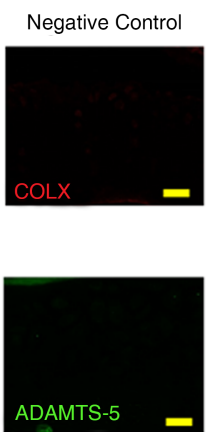

Figure 3 Inhibition of Rac1 delayed osteoarthritis (OA) cartilage destruction. (A) Rac1 activity regulation by lentivirus demonstrated by Rac1 pull-down assay (Rac1 activity was elevated by CA-Rac1 virus and was downregulated by DN-Rac1 activity). (B) Regulation of Rac1 activity by intra-articular injection of GFP, CA-Rac1 and DN-Rac1 lentivirus controlled OA cartilage destruction demonstrated by Safranin Orange staining and genes expression demonstrated by immunofluorescence. Scale bars, 200 or 50 or $30 \mu \mathrm{m}$. (CA-Rac1 group mice joints displayed the most severe $0 A$ phenotypes, such as cartilage damage or even loss, reduced Safranin 0 staining, increased ADAMTS-5 and COLX expression. However, DN-Rac1 showed great protective effect on cartilage.) (C) OARSI Scoring of OA severity upon lentivirus treatment. Values are means $\pm S D(n=3)$. ${ }^{*} p<0.05$, ${ }^{* *} p<0.01$. (D) Safranin 0 staining of normal cartilage. (E) NSC23766 greatly inhibited Rac1 activity. (F) Safranin 0 staining of cartilage sections in mice injected with NSC23766 and PBS. Scale bars, 200 or $50 \mu \mathrm{m}$. (PBS group mice cartilage showed cartilage clefts and reduced Safranin 0 staining, while NSC23766 could to a great extent protect cartilage from damage.) (G) OARSI Scoring of OA severity upon NSC23766 treatment. Values are means $\pm S D(n=3)$. ${ }^{*} p<0.05,{ }^{*} p<0.01$. ( $H$ and I) Immunofluorescence assay of COLX (H) and ADAMTS-5 (I) expression in cartilage upon NSC23766 administration. Scale bar, $30 \mu \mathrm{m}$. 
normal cartilage (figure 3D). Interestingly, intra-articular injection of CA-Rac1 lentivirus accelerated OA progression characterised by cartilage degradation, decreased Safranin Orange staining and elevated ADAMTS-5 and COLX expression. Consistently, the ectopic expression of DN-Rac1 in articular cartilage chondrocytes significantly reduced articular cartilage degradation and ADAMTS-5 and COLX expression (figure 3B). The OARSI score results showed that the DN-Rac1 group mice attained the lowest average score of 4.4 , while the CA-Rac1 group mice attained the highest average score of 19.5 (figure 3C). Furthermore, CA-Rac1 group mice displayed complete disorganisation or even loss of cartilage structure.

To further confirm the results, we investigated the effect of Rac1 inhibitor NSC23766 on regulating OA cartilage degradation. After 10 days post-OA surgery, NSC23766 dissolved in PBS was injected intra-articularly three times a week, and joints were collected at 4, 6 and 8 weeks postsurgery. Pull-down assay showed that Rac1 activity was successfully decreased upon NSC23766 administration (figure 3E). The representative photographs of the joint showed that NSC23766 administration prevented the formation of tibial cartilage lesions as shown by increased Safranin Orange staining and decreased OARSI score (figure 3F,G and figure S2). Additionally, expression levels of COLX and ADAMTS-5 were correspondingly decreased in chondrocytes of degenerating cartilage upon NSC23766 injection (figure 3H,I). Hence, our in vivo experiments clearly revealed the therapeutic effects of Rac1 inhibition on OA cartilage degeneration in mice.

\section{Rac1 regulates pathological changes in OA chondrocytes partially through the $\boldsymbol{\beta}$-catenin pathway}

In light of these data, we set out to uncover the underlying mechanisms of how activated Rac1 regulates MMP13, ADAMTS-5 and COLX expression. Rac1 has been recently demonstrated to regulate $\beta$-catenin nuclear translocation in canonical Wnt signalling. ${ }^{12}$ And activation of $\beta$-catenin in articular cartilage leads to OA-like phenotype characterised by upregulation of hypertrophy-related genes MMP9, MMP13, ALP, OCN and COLX. ${ }^{14}$ As shown in figure $4 \mathrm{~A}, \beta$-catenin nuclear accumulation is promoted by CA-Rac1 and impeded by DN-Rac1 in chondrocytes, which correlated with the study of Wu et $a l^{12}$ Additionally, Rac1 activation would further lead to the upregulation of the downstream factors Axin2 and LEF-1 (figure 4B). We therefore hypothesised that activated Rac1 might induce $\beta$-catenin nuclear accumulation and subsequently enhance MMP13, ADAMTS-5 and COLX expression. To verify this hypothesis, we block $\beta$-catenin nuclear accumulation and check if Rac1-induced OA phenotype can be affected. Since phosphorylation sites Ser191 and Ser605 of $\beta$-catenin are key residues for its nuclear localisation, ${ }^{12}$ we co-transfected $\beta$-catenin triple mutant (S191A, S246A, S605A) and Rac1, and checked whether they had a synergistic effect. As shown in figure 4C-E, co-expression of a $\beta$-catenin triple mutant with CA-Rac1 considerably abrogated CA-Rac1 induced MMP13, ADAMTS-5 and COLX upregulation (figure 4C-E). Furthermore, this $\beta$-catenin triple mutant reinforced the effect of DN-Rac1 on MMP13 and ADAMTS-5 expression (figure 4C-E). Taken together, these data suggested that Rac1 induced MMP13, ADAMTS-5 and COLX expression, partially through the $\beta$-catenin pathway.

\section{OA treatment using a cocktail of hyaluronic acid containing chitosan microspheres encapsulating NSC23766}

Rac1 inhibitor NSC23766 displayed satisfactory therapeutic effects on the mouse OA model. However, it is usually difficult to maintain an effective concentration of drugs, which hinders their further application. In order to elicit a sustaining effect, a strategy for controlled release of NSC23766 should be developed. Chitosan microsphere represents a useful tool for modified drug delivery as their preparation is quite easy and is useful for controlled drug release. In this study, we applied chitosan microspheres to encapsulate Rac1 inhibitor NSC23766 for controlled release in mice OA joints. The chitosan microspheres were spherical in shape with a mean size of around $100 \mu \mathrm{m}$, and had a smooth surface without cracks and winkles, as
A
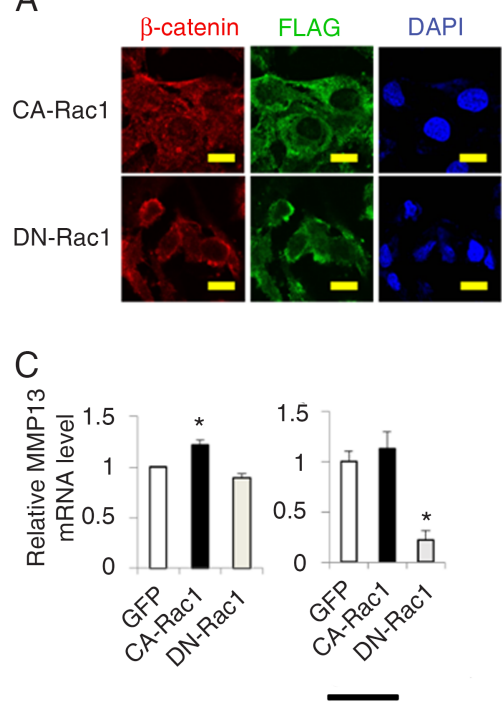

$\beta$-catenin tri-mutant
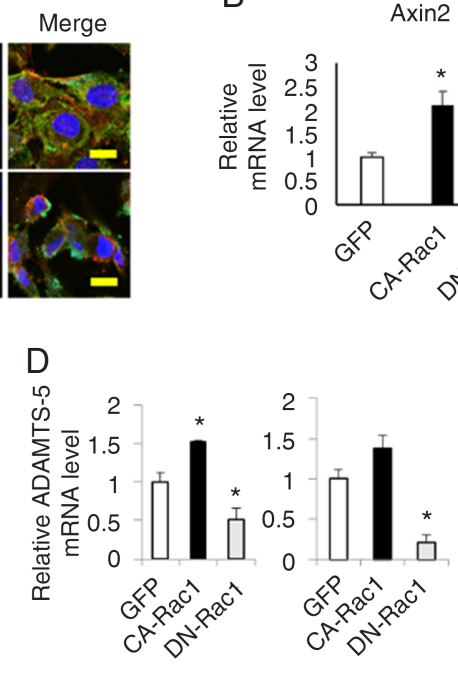

$\beta$-catenin tri-mutant
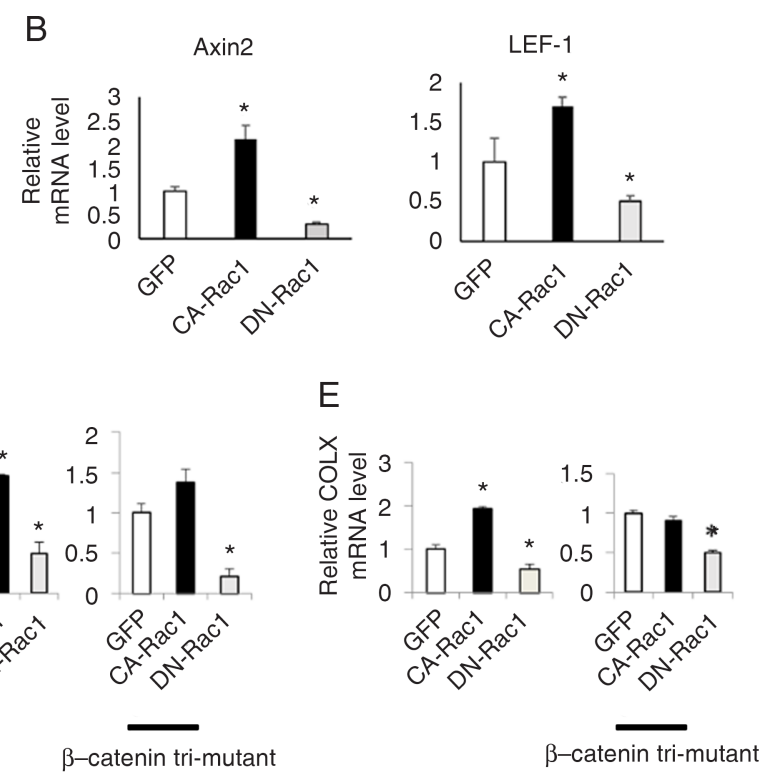

Figure 4 Rac1 induced chondrocytes pathologic change partially through regulation of $\beta$-catenin nuclear translocalisation. (A) $\beta$-catenin was enriched in nuclear in chondrocytes transfected with CA-Rac1, while mainly localised in cytoplasm and on cell membrane in chondrocytes transfected with DN-Rac1. (B) Rac1 activation resulted in Wnt downstream signalling Axin2 and LEF-1 activation. (C-E) $\beta$-catenin mutant (S191A, S246A, S605A) partially blocked CA-Rac1-induced MMP13 (C), ADAMTS-5 upregulation. (D) while completely blocked COLX (E) upregulation. Also, $\beta$-catenin mutant (S191A, S246A, S605A) strengthen the effect of DN-Rac1 on MMP13 (C), ADAMTS-5 (D) and COLX (E). Values are means \pm SD $(n=3) .{ }^{*} p<0.05,{ }^{* *} p<0.01$. 
demonstrated by scanning electron micrographs (figure 5A). The controlled release capacity of chitosan microspheres were determined by measuring the OD value of the extracts at $360 \mathrm{~nm}$ (which is specific for NSC23766). NSC23766 was released from microspheres in a biphasic fashion, characterised by a fast release phase during the initial first day followed by slower release on remaining days (figure 5B,C). HA containing chitosan microspheres encapsulating NSC23766 was injected into the OA joints once a week after 10 days postsurgery, and subjects were then analysed by histological examination (figure 5D). Histological examinations were made using Safranin Orange staining to visualise the time course of OA development. Joints in each group were also collected for the Rac1 pull-down assay to verify the inhibitory effect of NSC23766 (figure 5E). When joints were set $\mathrm{OA}$ model and further treated with chitosan microspheres containing NSC23766, they displayed markedly decreased cartilage destruction, more Safranin Orange staining and a lower OARSI score compared to joints treated with chitosan microspheres with PBS (control group), thus indicating a delay in OA development (figure 5F,G). Additionally, controlled release of NSC23766 in the joint resulted in decreased expression of chondrocyte hypertrophy marker COLX and matrix degradation gene ADAMTS-5 (figure 5F and figure S3).

In this study, we detected high Rac1 activity in osteoarthritic chondrocytes and demonstrated that aberrant Rac1 activation enhances chondrocyte hypertrophy and matrix degradationrelated gene expression. Inhibition of Rac1 via either DN-Rac1 lentivirus infection or Rac1 inhibitor NSC23766 delays experimentally induced OA.
A
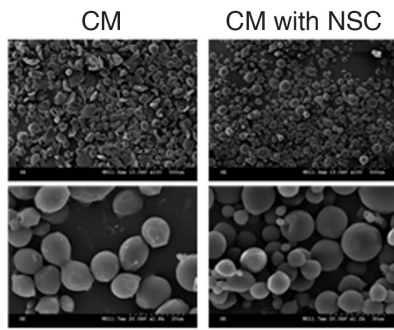

C

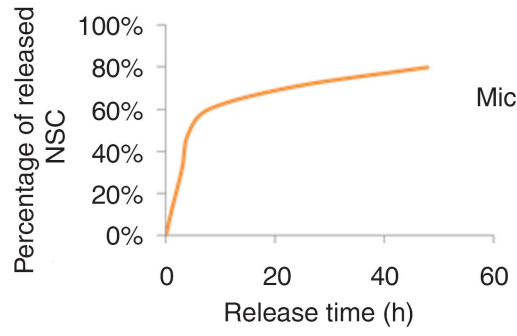

D

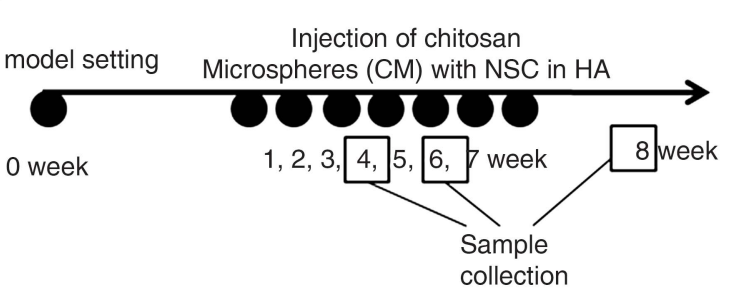

$\mathrm{F}$

CM

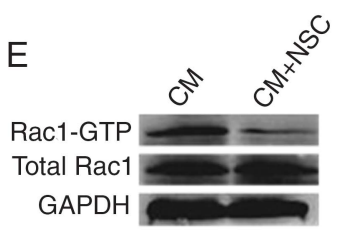

G
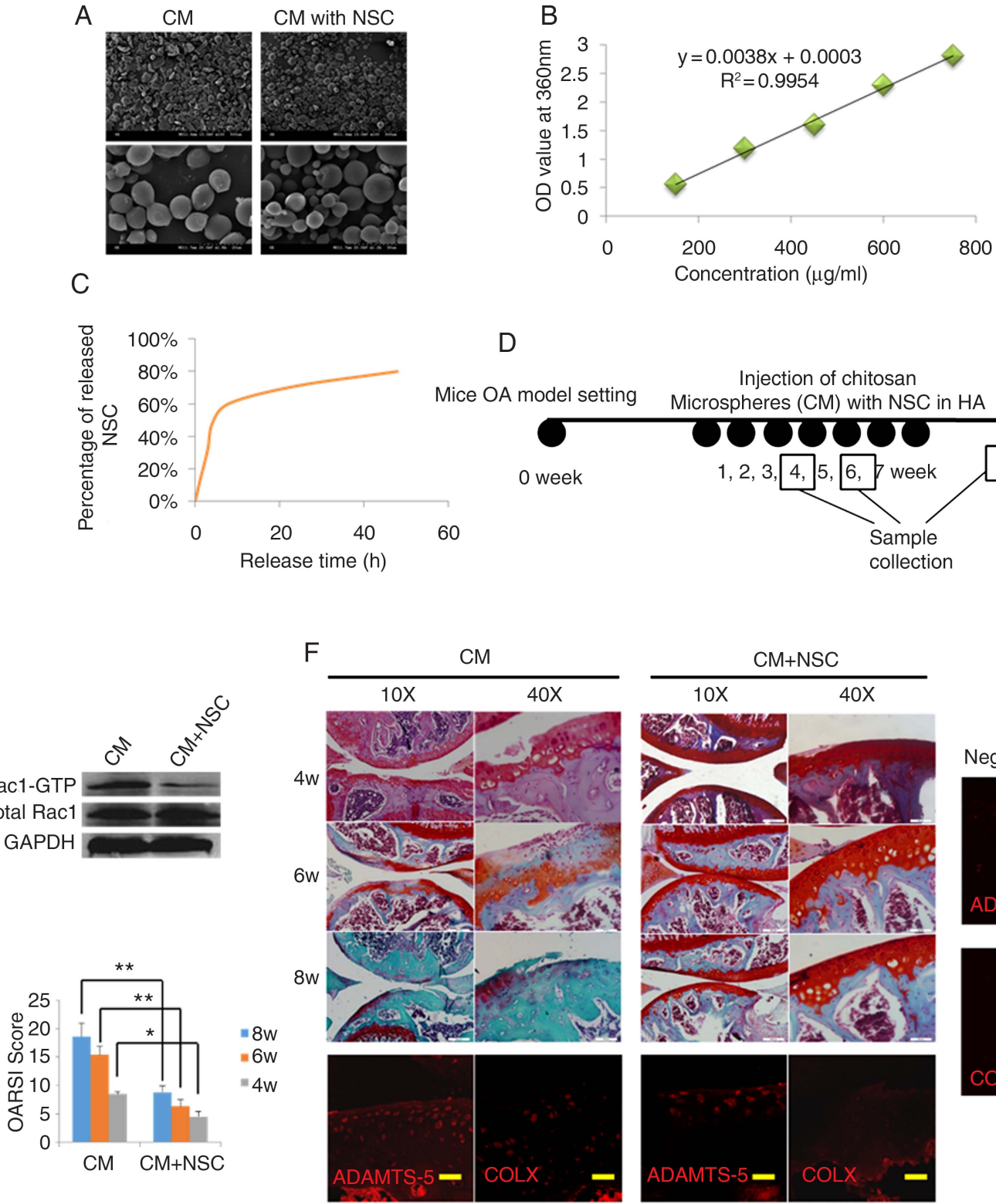

Negative Control

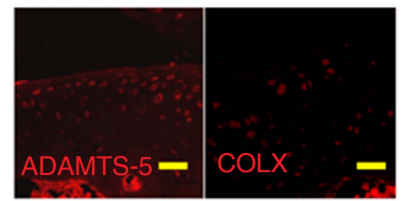

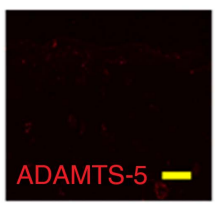

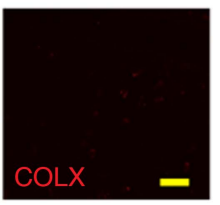

Figure 5 Therapeutic effect of hyaluronic acid containing chitosan microspheres encapsulating NSC23766 on mice osteoarthritis (OA). (A) Scanning electron micrographs of chitosan microspheres. (B) Standard curve of NSC23766. (C) The control release ability of chitosan microspheres.

(D) Timeline of experiment process. (E) Control release of NSC23766 greatly inhibited cartilage chondrocytes Rac1 activity (F). Regulation of Rac1 activity by hyaluronic acid containing chitosan microspheres encapsulating NSC23766 controlled OA cartilage destruction demonstrated by Safranin Orange staining and genes expression. Scale bars, 200 or 50 or $30 \mu \mathrm{m}$. (G) OARSI scoring of OA severity upon hyaluronic acid containing chitosan microspheres encapsulating NSC23766 treatment. Values are means \pm SD $(n=3)$. ${ }^{*} p<0.05,{ }^{* *} p<0.01$. 


\section{DISCUSSION}

This study, for the first time, illustrated the important role of aberrant activation of Rac1 in OA progression, that is, activation of Rac1-accelerated OA development, while inhibition of Rac1 protected cartilage from OA. It was known that there are 20 members of the Rho family of GTPases, which are 'Ras-like' proteins. Among these, Cdc42, Rac1 and RhoA have been most intensively studied. Rac1, Cdc42 and RhoA are all expressed in articular and growth plate chondrocytes, but they have distinct functions. Rac1 and Cdc42 accelerate chondrocyte differentiation while RhoA/ROCK signalling exerts an antagonistic effect on chondrocyte development. ${ }^{8} 9$ Here, we found that Rac1 could be activated by the proinflammatory factor IL1 $\beta$ and that Rac1 activation resulted in upregulation of MMP13, ADAMTS-5, COLX and Runx2 expression. This is consistent with the recent in vitro study of Long and colleagues, ${ }^{13}$ which demonstrated that Rac1 is required for chondrocyte MMP13 production. More importantly, our in vivo study provided solid evidence to confirm that inhibition of Rac1 activity in articular chondrocytes delayed OA development, as evidenced by reduced ADAMTS-5 and COLX expression and cartilage degradation. It therefore remains an exciting prospect to determine the upstream signalling proteins implicated in modulating Rac1 activity in OA development.

However, in this study, the joints of the CA-Rac1 group mice displayed exceedingly severe detrimental outcome, with both surface cartilage and subchondral bone being lost. This observed effect might be explained as follows. As Rac1 upregulates ADAMTS-5 and MMP13, the exceedingly high activity of Rac1 overexpressed by the CA-Rac1 virus might lead to extensive cartilage degradation, resulting in complete loss during a short time duration. This, in turn, further aggravates osteochondral bone damage under mechanical stress after 6 weeks. Therefore, the high activity of Rac1 amplifies the effects of pathological factors on chondrocytes in the OA model induced by anterior cruciate ligament transection.

The Wnt- $\beta$-catenin pathway has been implicated not only in the embryogenesis of the joint but also in adult skeletal homeostasis. ${ }^{15-18}$ Conditional activation of $\beta$-catenin in articular chondrocytes predisposes adult mice to OA-phenotype, characterised by accelerated chondrocyte maturation and osteophyte formation. ${ }^{14}$ Rho GTPases are believed to play a significant role in cytoplasmic $\beta$-catenin nuclear transportation in the canonical Wnt signalling pathway. ${ }^{19}$ Among these, Rac1 was reported to cooperate with JNK2 in $\beta$-catenin phosphorylation and nuclear localisation. ${ }^{12}$ Rac1 ablation phenocopies $\beta$-catenin removal in limb development. ${ }^{12}$ Our data, for the first time, demonstrated that in chondrocytes, $\beta$-catenin might partially function downstream of activated Rac1 in modulating pathological changes in chondrocytes. It is of interest to investigate whether Rac1 could directly interact with $\beta$-catenin and trigger its cellular translocation. In our study, mutated $\beta$-catenin could only partially block CA-Rac1-induced ADAMTS-5 and MMP13 upregulation, which suggests that other pathways independent of the $\beta$-catenin pathway might also be involved. This might also explain why in vivo CA-Rac1 virus injection led to a much more severe OA outcome. On the other hand, as Rho GTPases are 'molecular switches' for transferring signals from chondrocyte extracellular matrix to downstream effectors, ${ }^{20}$ Rac1 may induce pathological changes to chondrocytes via its downstream effectors. Also, it is not clear whether Cdc42 and RhoA/ROCK signalling exerts similar or adverse influence on OA pathology. All these possibilities await further detailed studies.
Inhibition of Rac1 exhibited therapeutic effect on OA treatment. This warrants further investigation on developing a strategy of local delivery of Rac1 inhibitor for OA treatment. Local delivery of reagents will be more effective at targeting with less accompanying side effects. Chitosan microspheres have potential application in drug delivery systems because they are a cheap, biocompatible, biodegradable and non-toxic natural polymer that enables the controlled release of many drugs. ${ }^{21-24}$ $\mathrm{HA}$ is an anionic, non-sulfated glycosaminoglycan distributed widely throughout connective, epithelial and neural tissues. It is one of the chief components of articular cartilage and has been used to treat OA of the knee via injection into the joint. ${ }^{25-27}$ In this study, HA containing chitosan microspheres encapsulated Rac1 inhibitor NSC23766 was injected into OA knees, to enable sustained release as well as lubrication of the joints. The cocktail of HA and biomaterial encapsulated NSC23766 may function as a potential strategy for OA therapy. However, the controlled release system used in this study only guarantees sustained release for 3 days. Nevertheless, the results were very encouraging and supportive of the local delivery strategy. Further studies will aim to develop more efficient delivery systems by controlling size and homogeneity of biomaterial spheres to maintain drug concentration in the OA joint.

In conclusion, our results indicate that Rac1 acts as a crucial mediator of $\mathrm{OA}$ cartilage destruction, by regulating catabolic factors, such as MMP13 and ADAMTS-5, and hypertrophyrelated factors, such as COLX and Runx2. Moreover, our work provides a new therapeutic strategy for OA treatment through inhibition of the aberrant activation of Rac1 with bioengineering technology.

Acknowledgements We would like to thank Huang Lu and Cai Youzhi for providing the human $\mathrm{OA}$ cartilage samples from patients undergoing total knee replacement. Additionally, we also thank Professor Wu Ximei for providing the $\beta$-catenin mutants.

Contributors SZ: performed research, analysed data, wrote the manuscript. $\mathrm{PL}, \mathrm{HL}$, PC and YW: analysed data, discussion of results. SH, QX and XZ: discussion of results. BCH: manuscript correction. YZ, HWO: Design research, manuscript correction.

Funding This work was supported by the National key scientific research projects (2012CB966604), the National Natural Science Fund (81125014, 81101356, $81201395, \mathrm{J1103603}, 31000436)$, Zhejiang province public welfare fund (2012C3112, 2012C33015), Zhejiang Provincial Natural Science Foundation of China (LY13C100001), the National High Technology Research and Development Program of China (2012AA020503), Postdoctoral Foundation of China (2013M531465), Postdoctoral Foundation of Zhejiang Province (BSH1302078), as well as sponsored by the Regenerative Medicine in Innovative Medical Subjects of Zhejiang Province and Zhejiang Provincial Program for the Cultivation of High-level Innovative Health Talents.

\section{Competing interests None.}

\section{Patient consent Obtained.}

Ethics approval Zhejiang University Ethics Committee.

Provenance and peer review Not commissioned; externally peer reviewed.

Open Access This is an Open Access article distributed in accordance with the Creative Commons Attribution Non Commercial (CC BY-NC 3.0) license, which permits others to distribute, remix, adapt, build upon this work non-commercially, and license their derivative works on different terms, provided the original work is properly cited and the use is non-commercial. See: http://creativecommons.org/ licenses/by-nc/3.0/

\section{REFERENCES}

1 der Mark KV, Kirsch T, Nerlich A, et al. Type X collagen synthesis in human osteoarthritic cartilage. Indication of chondrocyte hypertrophy. Arthritis Rheum 1992;35:806-11.

2 Zheng Q, Zhou G, Morello R, et al. Type X collagen gene regulation by Runx2 contributes directly to its hypertrophic chondrocyte-specific expression in vivo. J Cell Biol 2003; 162:833-42. 
3 Shlopov BV, Gumanovskaya ML, Hasty KA. Autocrine regulation of collagenase 3 (matrix metalloproteinase 13) during osteoarthritis. Arthritis Rheum 2000;43:195-205.

4 Majumdar MK, Askew R, Schelling S, et al. Double-knockout of ADAMTS-4 and ADAMTS- 5 in mice results in physiologically normal animals and prevents the progression of osteoarthritis. Arthritis Rheum 2007;56:3670-4.

5 Stanton $\mathrm{H}$, Rogerson FM, East CJ, et al. ADAMTS5 is the major aggrecanase in mouse cartilage in vivo and in vitro. Nature 2005;434:648-52.

6 Kamekura S, Kawasaki Y, Hoshi K, et al. Contribution of runt-related transcription factor 2 to the pathogenesis of osteoarthritis in mice after induction of knee joint instability. Arthritis Rheum 2006;54:2462-70.

7 Woods A, Wang G, Dupuis $\mathrm{H}$, et al. Rac1 signaling stimulates $\mathrm{N}$-cadherin expression, mesenchymal condensation, and chondrogenesis. J Biol Chem 2007:282:23500-8.

8 Wang G, Beier F. Rac1/Cdc42 and RhoA GTPases antagonistically regulate chondrocyte proliferation, hypertrophy, and apoptosis. J Bone Miner Res 2005;20:1022-31.

9 Wang $\mathrm{G}$, Woods A, Agoston $\mathrm{H}$, et al. Genetic ablation of Rac1 in cartilage results in chondrodysplasia. Dev Biol 2007;306:612-23.

10 Wang G, Yan Q, Woods A, et al. Inducible nitric oxide synthase-nitric oxide signaling mediates the mitogenic activity of Rac1 during endochondral bone growth. J Cell Sci 2011;124(Pt 20):3405-13.

11 Suzuki D, Yamada A, Amano T, et al. Essential mesenchymal role of small GTPase Rac1 in interdigital programmed cell death during limb development. Dev Biol 2009;335:396-406.

12 Wu X, Tu X, Joeng KS, et al. Rac1 activation controls nuclear localization of beta-catenin during canonical Wnt signaling. Cell 2008;133:340-53.

13 Long DL, Willey JS, Loeser RF. Rac1 is required for matrix metalloproteinase-13 production by chondrocytes in response to fibronectin fragments. Arthritis Rheum 2013;65:1561-8.

14 Zhu M, Tang D, Wu Q, et al. Activation of beta-catenin signaling in articular chondrocytes leads to osteoarthritis-like phenotype in adult beta-catenin conditional activation mice. J Bone Miner Res 2009;24:12-21.
15 Hartmann C, Tabin CJ. Wnt-14 plays a pivotal role in inducing synovial joint formation in the developing appendicular skeleton. Cell 2001;104:341-51.

16 Guo X, Day TF, Jiang X, et al. Wnt/beta-catenin signaling is sufficient and necessary for synovial joint formation. Genes Dev 2004;18:2404-17.

17 Hartmann C, Tabin CJ. Dual roles of Wnt signaling during chondrogenesis in the chicken limb. Development 2000;127:3141-59.

18 Boyden LM, Mao J, Belsky J, et al. High bone density due to a mutation in LDL-receptor-related protein 5. N Engl J Med 2002:346:1513-21.

19 Schlessinger K, Hall A, Tolwinski N. Wnt signaling pathways meet Rho GTPases. Genes Dev 2009;23:265-77.

20 Etienne-Manneville $S$, Hall A. Rho GTPases in cell biology. Nature 2002;420:629-35.

21 Alpar HO, Somavarapu S, Atuah KN, et al. Biodegradable mucoadhesive particulates for nasal and pulmonary antigen and DNA delivery. Adv Drug Deliv Rev 2005; $57: 411-30$

22 Illum L, Watts P, Fisher AN, et al. Intranasal delivery of morphine. J Pharmacol Exp Ther 2002;301:391-400.

23 Jain SK, Chourasia MK, Jain AK, et al. Development and characterization of mucoadhesive microspheres bearing salbutamol for nasal delivery. Drug Deliv 2004;11:113-22

24 Kang ML, Kang SG, Jiang HL, et al. In vivo induction of mucosal immune responses by intranasal administration of chitosan microspheres containing Bordetella bronchiseptica DNT. Eur J Pharm Biopharm 2006;63:215-20.

25 Lo GH, LaValley M, McAlindon T, et al. Intra-articular hyaluronic acid in treatment of knee osteoarthritis: a meta-analysis. JAMA 2003;290:3115-21.

26 Munteanu SE, Zammit GV, Menz HB, et al. Effectiveness of intra-articular hyaluronan (Synvisc, hylan G-F 20) for the treatment of first metatarsophalangeal joint osteoarthritis: a randomised placebo-controlled trial. Ann Rheum Dis 2011;70:1838-41.

27 Yatabe T, Mochizuki S, Takizawa M, et al. Hyaluronan inhibits expression of ADAMTS4 (aggrecanase-1) in human osteoarthritic chondrocytes. Ann Rheum Dis 2009;68:1051-8. 